PROCEEDINGS OF THE

AMERICAN MATHEMATICAL SOCIETY

Volume 129, Number 3, Pages 749-751

S 0002-9939(00)05617-3

Article electronically published on September 19, 2000

\title{
ALMOST-EVERYWHERE DISCONTINUITY OF THE SPECTRAL RADIUS
}

\author{
THOMAS J. RANSFORD
}

(Communicated by Joseph A. Ball)

\begin{abstract}
Let $\rho(T)$ denote the spectral radius of an operator $T$. We construct operators $S$ and $T$ on $\ell^{2}$ such that $\lambda \mapsto \rho(T-\lambda S)$ is discontinuous almost everywhere on the unit disk.
\end{abstract}

Let $X$ be a complex Banach space, and denote by $\mathcal{B}(X)$ the algebra of bounded linear operators on $X$. Given $T \in \mathcal{B}(X)$, its spectral radius $\rho(T)$ can be calculated via the Beurling-Gelfand formula:

$$
\rho(T)=\lim _{n \rightarrow \infty}\left\|T^{n}\right\|^{1 / n}=\inf _{n \geq 1}\left\|T^{n}\right\|^{1 / n} .
$$

From the second equality, it follows easily that the map $T \mapsto \rho(T)$ is upper semicontinuous on $\mathcal{B}(X)$. However, in general it need not be continuous. Kakutani gave an example (first published in $\left[\mathrm{R}\right.$, pp. 282-283]) of a sequence $\left(T_{n}\right)$ of nilpotent operators on $\ell^{2}$ converging to an operator $T$ with $\rho(T)>0$. Müller [M] subsequently refined Kakutani's example to produce a pair of operators $S, T$ on $\ell^{2}$ such that the map $\lambda \mapsto \rho(T-\lambda S)$ is discontinuous at 0 . Thus even the restriction of $\rho$ to a one-dimensional affine subspace can be discontinuous. (These constructions are also described in detail in the book of Aupetit [A. Chapitre 1, §5], which further contains interesting examples of spectral discontinuity in other Banach algebras.)

Müller's example is combinatorial in nature. The purpose of this note is to show how, using a little elementary potential theory, it is possible to circumvent most of the combinatorial intricacies in [M], and at the same time obtain the following stronger result.

Theorem. There exist $S, T \in \mathcal{B}\left(\ell^{2}\right)$ such that the map $\lambda \mapsto \rho(T-\lambda S)$ is discontinuous at almost every point of the unit disk.

The combinatorics are replaced by the following simple lemma.

Lemma. Let $X$ be a complex Banach space, and let $S, T \in \mathcal{B}(X)$ with $\rho(S)>0$. Then $\rho(T-\lambda S)>0$ for all $\lambda \in \mathbf{C}$ outside a set of Lebesgue measure zero.

Received by the editors May 4, 1999.

2000 Mathematics Subject Classification. Primary 47A11.

The author's research was supported by grants from NSERC (Canada) and the Fonds FCAR (Québec). 
Proof of the Lemma. By Vesentini's theorem (see e.g. [A, Chapitre 1, §2, Théorème 1]), the function $z \mapsto \log \rho(S+z T)$ is subharmonic. In particular, for each $r>0$,

$$
\frac{1}{2 \pi} \int_{0}^{2 \pi} \log \rho\left(S+r e^{i \theta} T\right) d \theta \geq \log \rho(S) .
$$

Multiplying by $r$ and integrating from $r=0$ to $R$ gives

$$
\frac{1}{2 \pi} \int_{|z| \leq R} \log \rho(S+z T) d m(z) \geq \frac{1}{2} R^{2} \log \rho(S)>-\infty
$$

where $m$ denotes two-dimensional Lebesgue measure. In particular, $\rho(S+z T)>0$ almost everywhere on $|z| \leq R$. As $R$ is arbitrary, $\rho(S+z T)>0$ for almost every $z \in$ C. Hence, finally, $\rho(T-\lambda S)=|\lambda| \rho(S+(-1 / \lambda) T)>0$ for almost every $\lambda \in \mathbf{C}$.

Proof of the Theorem. Let $E$ be a countable dense subset of the closed unit disk, say $E=\left\{\alpha_{0}, \alpha_{1}, \alpha_{2}, \ldots\right\}$. Define a new sequence $\left(\beta_{n}\right)_{n \geq 1}$ by setting $\beta_{n}=\alpha_{m}$, where $m$ is the largest integer such that $2^{m}$ divides $n$. Thus

$$
\left(\beta_{1}, \beta_{2}, \ldots\right)=\left(\alpha_{0}, \alpha_{1}, \alpha_{0}, \alpha_{2}, \alpha_{0}, \alpha_{1}, \ldots\right) .
$$

The sequence $\left(\beta_{n}\right)$ has the property that every set of $2^{m+1}$ consecutive terms contains $\alpha_{m}$.

We are now ready to define the operators $S$ and $T$. Writing $\left(e_{n}\right)_{n \geq 1}$ for the standard unit vector basis of $\ell^{2}$, we let $S$ be the standard right shift $S e_{n}=e_{n+1}$, and $T$ be the weighted right shift $T e_{n}=\beta_{n} e_{n+1}$. Note that $T-\lambda S$ is then also a weighted shift, and

$$
(T-\lambda S)^{k} e_{n}=\left(\beta_{n}-\lambda\right)\left(\beta_{n+1}-\lambda\right) \cdots\left(\beta_{n+k-1}-\lambda\right) e_{n+k} .
$$

As every set of $2^{m+1}$ consecutive $\beta_{n}$ contains $\alpha_{m}$, it follows that $\left(T-\alpha_{m} S\right)^{2^{m+1}}=0$. Hence $\rho(T-\lambda S)=0$ for all $\lambda \in E$. Since $E$ is dense in the unit disk, it follows that $\lambda \mapsto \rho(T-\lambda S)$ is discontinuous at each $\lambda$ in the disk where $\rho(T-\lambda S)>0$. But from the lemma, $\rho(T-\lambda S)>0$ almost everywhere.

Remarks. (1) With a little more potential theory, this result can be strengthened. Indeed, by the classical theorem of Cartan, a subharmonic function on $\mathbf{C}$ which is not identically $-\infty$ can only take the value $-\infty$ on a set of capacity zero (see e.g. [HK Theorem 5.32]). Applying this to the function $\lambda \mapsto \log (T-\lambda S)$, we see that 'Lebesgue measure zero' can be replaced by 'capacity zero' in the lemma, and hence also in the theorem. On the other hand, it is not possible to go the whole way and find operators $S, T$ for which $\lambda \mapsto \rho(T-\lambda S)$ is discontinuous everywhere on the unit disk. This is because an upper semicontinuous function on a complete metric space always has a dense $G_{\delta}$ set of points of continuity (see e.g. [K] $§ 39$, IV.2]).

(2) The operators $S$ and $T$ were defined on $\ell^{2}$ for simplicity, but exactly the same construction works on any complex Banach space $X$ with an (infinite) symmetric basis (see e.g. [LT, Chapter 3]). Thus, for every such $X$, the spectral radius function $\rho$ is discontinuous. On the other hand, there exist infinite-dimensional Banach spaces for which $\rho$ is continuous. For example, if $X$ is the hereditarily indecomposable space constructed in $\overline{\mathrm{GM}}$, $\S 3]$, then every $T \in \mathcal{B}(X)$ has countable spectrum [GM, Theorem 18], and by Newburgh's theorem (see e.g. [A, Chapitre 1, $\S 1$, Corollaire 7]), this is sufficient to imply that $\rho$ be continuous. The exact determination of those spaces for which $\rho$ is continuous remains a challenging open problem. 


\section{REFERENCES}

[A] B. Aupetit, Propriétés spectrales des algèbres de Banach, Lecture Notes in Mathematics 735, Springer-Verlag, Berlin, 1979. MR 81i:46055

[GM] W. T. Gowers and B. Maurey, The unconditional basic sequence problem, J. Amer. Math. Soc. 6 (1993), 851-874. MR 94k:46021

[HK] W. Hayman and P. B. Kennedy, Subharmonic Functions vol 1, Academic Press, London, 1976. MR 57:665

[K] K. Kuratowski, Topology vol II, PWN Polish Scientific Publishers, Warsaw, 1968. MR 41:4467

[LT] J. Lindenstrauss and L. Tzafriri, Classical Banach Spaces vol I, Springer-Verlag, Berlin, 1977. MR 58:17766

[M] V. Müller, On discontinuity of the spectral radius in Banach algebras, Comment. Math. Univ. Carolinae 17 (1976), 591-598. MR 58:17847

[R] C. E. Rickart, General Theory of Banach Algebras, Robert E. Krieger, New York, 1974. MR 22:5903

Département de Mathématiques et de Statistique, Université Laval, Québec, Québec, CANADA G1K 7P4

E-mail address: ransford@mat.ulaval.ca 